\title{
Homogeneous and Localized Deformation in Poly(Methyl Methacrylate) Nanocutting
}

\author{
Fengzhen Sun ${ }^{1,2}$ (D) E. Kristofer Gamstedt ${ }^{1}$
}

Received: 17 September 2018 / Revised: 7 December 2018 / Accepted: 17 December 2018 / Published online: 1 February 2019

(c) The Author(s) 2019

\begin{abstract}
Nanoscale manufacturing imposes demands on prediction of cutting processes on small scales. Predictive modeling schemes based on the underlying physical mechanisms could potentially be more generally applicable in manufacturing. In this work, the experimental and numerical studies on polymethyl methacrylate (PMMA) nanocutting are reported. The cutting experiments were performed on an ultramicrotome instrumented with piezoelectric transducers to measure the cutting forces on cutting down to about $60 \mathrm{~nm}$ thickness. Using atomic force microscopy, the surface damage was identified as shear yield bands triggered by adiabatic heating. A suitable physical model including these observed phenomena made it possible to link the processing conditions with the onset of damage, i.e., the transition between a high-quality transparent surface and a damaged uneven surface. Finite element analysis was carried out to investigate the deformation modes of PMMA under different cutting conditions and to predict the formation of the undesired shear bands. From an engineering perspective, such an approach could be potentially useful in improving manufacturing control.
\end{abstract}

Keywords Nanocutting $\cdot$ Shear band $\cdot$ Adiabatic shearing $\cdot$ Deformation mode

\section{Introduction}

The fast development in device miniaturization demands increased abilities to manipulate matter at nanoscale and even atomic level. Cutting of polymers at nanoscale (submicroscale) is of great significance in manufacturing components and devices for electrical and optical applications [1]. As cutting goes down to small scales, the mechanical mechanisms related to material volume (e.g., the plastic work) are increasingly restricted, while the mechanisms related to surface area (e.g., adhesion, fracture) become relatively more significant [2].

Polymers can deform plastically, with chain molecules sliding past each other over relatively large distances. The plastic behavior of amorphous polymers strongly depends on the temperature and deformation rate [3]. Far below the glass transition temperature $T_{\mathrm{g}}$, chain molecules cannot easily

Fengzhen Sun

f.sun@imperial.ac.uk

1 Department of Engineering Sciences, Uppsala University, 75121 Uppsala, Sweden

2 Department of Mechanical Engineering, Imperial College London, London SW7 2AZ, UK slide past each other because the secondary bonds between molecules are tight. As polymers are subjected to external loading, brittle failure usually takes place by breaking the intermolecular bonds [4]. When the temperature approaches about $0.8 T_{\mathrm{g}}$, the molecules gain some mobility and polymers exhibit certain ductility. When the temperature approaches $T_{\mathrm{g}}$, the chain molecules become more mobile and can rearrange under loading. Under sufficient large deformation, molecular chains are drawn and orientated in parallel, leading to local hardening in the direction of loading. If the temperature exceeds $T_{\mathrm{g}}$, the molecules obtain very high mobility and polymers show relatively viscous behavior. Varying the strain rate also influences the deformation mode. Generally, increasing the strain rate, i.e., decreasing the testing time, is equivalent to decrease the temperature. However, increasing the strain rate may lead to the isothermal-adiabatic transition. Under high rate plastic deformation, the heat converted from the plastic work in polymer cannot dissipate to the surrounding regions rapidly due to the low thermal diffusivities $\left(\sim 10^{-7} \mathrm{~m}^{2} / \mathrm{s}\right.$, two orders of magnitude lower than metals [5]), and thus it results in thermal softening, which can lead to brittle-ductile deformation transition [6]. In addition, a glassy polymer exhibits apparent scale effects (or cube-square scaling effects) [2]; namely it tends to deform 
in a ductile manner at small scale while behaves in a brittle fashion at large scale. Since cutting is a shear-dominated dynamic process, glassy polymer may exhibit different deformation modes when the cutting speed or cutting thickness (depth of cut) varies, which directly influence the surface qualities in manufacturing of engineering applications.

Investigating the mechanical response of glassy polymers during cutting process is important for both scientific studies and engineering applications. The main objective of this study is to reveal the mechanisms that govern the deformation behavior of polymeric material during nanocutting by well-controlled experiments and modeling. Such knowledge can be useful in controlling and limiting damage formation in manufacturing of small-scale polymer components, and in models predicting the mechanical behavior on the submicrometer level in polymer-matrix composites.

\section{Nanocutting Experiments}

In order to have a complete structure and content, some experimental work and results already published in Refs [7] and [8] are briefly summarized in the beginning of this paper.

\subsection{Experiment Setup}

In order to assess the mechanical behavior of polymers during nanocutting process, an ultramicrotome instrumented with sensitive piezoelectric load sensors (PCB Piezotronics, 209A12) was set up, as shown in Fig. 1. This instrumented ultramicrotome can measure the cutting forces along and normal to the cutting directions. A diamond knife tool (Diatome MC3295, nominal edge radius of $7 \mathrm{~nm}$ ) attached with a water trough was used in this machine. The diamond knife was installed on the knife stage which can only move along the horizontal axis. The rake angle of the knife was set to

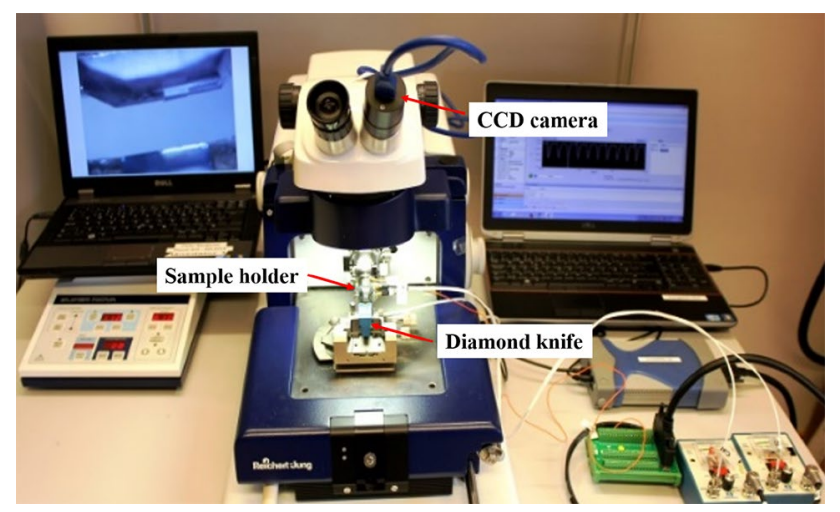

Fig. 1 An ultramicrotome instrumented with piezoelectric sensors for nanocutting test, adopted from [7] $40^{\circ}$. The ultramicrotome can advance the knife with a minimum of $5 \mathrm{~nm}$ and maximum of $200 \mathrm{~nm}$ distance by a thermal-feed control unit. A CCD eyepiece camera (ToupTek, S3CMOS) was installed on this ultramicrotome to provide high-quality images of the formed chips, which were used to determine the thickness of the obtained chips.

\subsection{Materials and Methods}

A commercial extruded polymethyl methacrylate (PMMA, $2 \mathrm{~mm}$ thickness, tensile strength of $70 \mathrm{MPa}$ and Young's modulus of $3.2 \mathrm{GPa}$ ) plate provided by Quinn Plastics was chosen for the nanocutting. PMMA was selected as a model material, since it is amorphous and widely used in optical and electronics applications. The PMMA samples were prepared according to the ultramicrotome manual.

Nanocutting at different thicknesses $\left(t_{\mathrm{u}}\right)$ and speeds $\left(v_{0}\right)$, as shown in Table 1, were carried out. After nanocutting, the cut surfaces of the work material were examined by atomic force microscopy (AFM, Bruker Multimode 8) in the ScanAsyst mode based on a peak force tapping mechanism, using a silicon tip with a radius of $3 \mathrm{~nm}$.

\section{Experimental Results}

This section presents the results of the two sets of nanocutting experiments described above in terms of the macroscopic features of the formed PMMA chips, the cut surfaces of the work material, and the mechanical behavior. More details about the experiments can be found in $[7,8]$.

\subsection{Nanocutting with Varying $t_{\mathrm{u}}$}

\subsubsection{Chip and Surface Morphologies}

Figure 2 displays the macroscopic features of PMMA chips formed at $t_{\mathrm{u}}=60 \mathrm{~nm}$ and $t_{\mathrm{u}}=140 \mathrm{~nm}$, which were taken through the CCD camera. It shows that the formed PMMA chips had integral shapes, which implies that the nanocutting was governed by a ductile deformation mechanism [2,9]. Figure 2 also indicates that microcracks were formed in the chips, orientating in the transverse direction.

Table 1 Experimental settings for cutting thickness and speed

\begin{tabular}{lll}
\hline & $t_{\mathrm{u}}(\mathrm{nm})$ & $v_{0}(\mathrm{~mm} / \mathrm{s})$ \\
\hline Varying $t_{\mathrm{u}}$ & $60,85,110,140,170,200$ & 1.0 \\
Varying $v_{0}$ & 85 & $0.25,0.5,1.0,3.0,10.0$ \\
\hline
\end{tabular}


Fig. 2 Macroscopic appearances of the formed PMMA chips, from [7]
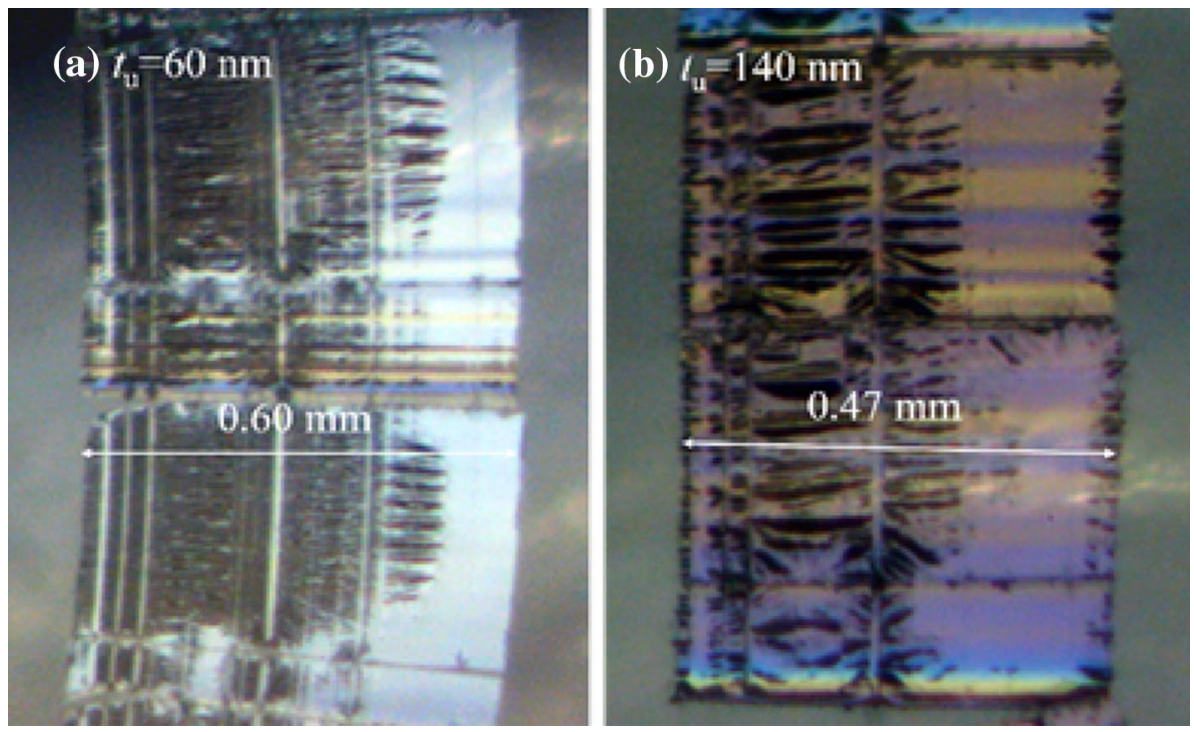

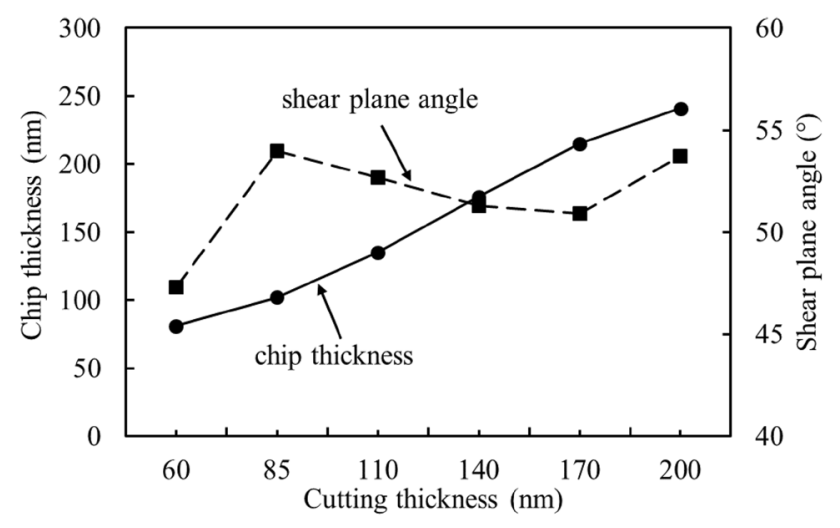

Fig. 3 Chip thickness and shear plane angle as a function of the cutting thickness

Due to the difficulty in quantifying the thickness of the nanoscale chips by experiment, the chip thickness is estimated as

$t_{\mathrm{c}}=\frac{w_{\mathrm{u}} l_{\mathrm{u}} t_{\mathrm{u}}}{w_{\mathrm{c}} l_{\mathrm{c}}}$ where $w_{\mathrm{u}}$ is the cutting width, $l_{\mathrm{u}}$ is the cutting length, $w_{\mathrm{c}}$ is the chip width, and $l_{\mathrm{c}}$ is the chip length that were immediately measured after nanocutting using the installed CCD camera. Therefore, the angle of the shear plane $\phi$ where plastic shearing mostly takes place can be obtained as

$\phi=\tan ^{-1}\left(\frac{t_{\mathrm{u}} \cos \alpha}{t_{\mathrm{c}}-t_{\mathrm{u}} \sin \alpha}\right)$

where $\alpha=40^{\circ}$ is the knife rake angle. The values of chip thickness and shear plane angle as a function of the cutting thickness are plotted in Fig. 3. It is seen that the chip thickness increases monotonically as the cutting thickness increases while the shear plane angle varies little for $t_{\mathrm{u}} \geq 85 \mathrm{~nm}$.

After nanocutting, the cut surfaces of the bulk PMMA were examined by AFM. A transition of the surface feature was found at a critical cutting thickness. Figure 4a displays the morphology of the surface cut at $t_{\mathrm{u}}=60 \mathrm{~nm}$, flat and smooth. As $t_{\mathrm{u}}$ increased to $85 \mathrm{~nm}$, short and weak wavelike features began to appear on the surface. As $t_{\mathrm{u}}$ increased
Fig. 4 AFM images of the cut surfaces created at $t_{\mathrm{u}}=60$ and $140 \mathrm{~nm}$, with arrows indicating the cutting directions
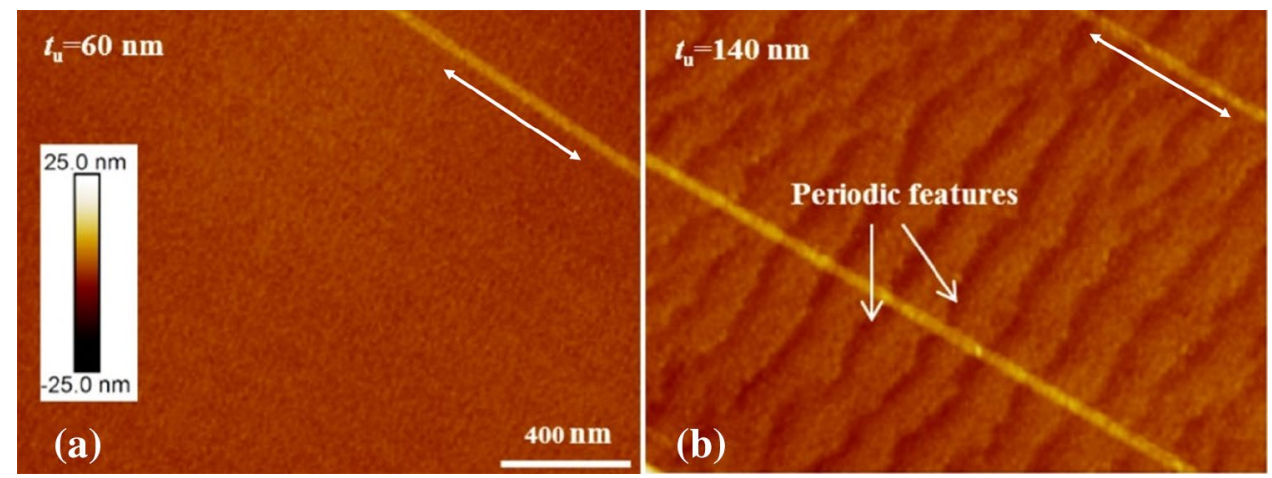
beyond $110 \mathrm{~nm}$, pronounced periodic features formed on the surfaces [7]. These features oriented in parallel and perpendicular to the cutting direction, as shown in Fig. 4b. The average spacing between adjacent features exhibited a linear dependence on the cutting thickness [7]. The features were similar to these in metals and polymer composites [10,11], which were explained by adiabatic shearing on the shear plane.

\subsubsection{Fracture Energy}

During cutting, two force components, the cutting force $F_{\mathrm{c}}$ and thrust force $F_{\mathrm{t}}$ which are, respectively, along and normal to the cutting direction, were collected by the load sensors instrumented in the sample holder (Table 2). The external work for cutting is mainly dissipated by plastic deformation, friction on the chip-knife interface and the fracture formation. The cutting force $F_{\mathrm{c}}$ satisfies the relation [12],

$\frac{F_{\mathrm{c}}}{w_{\mathrm{u}}}=\left(\frac{\tau_{y} \gamma}{Q}\right) t_{\mathrm{u}}+\frac{R}{Q}$

where $\tau_{y}$ is the flow stress of the material, $\gamma$ is the plastic strain, $Q=\{1-\sin \beta \sin \phi /[\cos (\beta-\alpha) \cos (\phi-\alpha)]\}$ is the friction parameter, $R$ is the fracture energy (work of surface

Table 2 Average values of cutting force $F_{\mathrm{c}}$ and thrust force $F_{\mathrm{t}}$ during nanocutting at different thicknesses, $v_{0}=1.0 \mathrm{~mm} / \mathrm{s}$

\begin{tabular}{lll}
\hline$t_{\mathrm{u}}(\mathrm{nm})$ & $F_{\mathrm{c}} / w_{\mathrm{u}}(\mathrm{N} / \mathrm{m})$ & $F_{\mathrm{r}} / w_{\mathrm{u}}(\mathrm{N} / \mathrm{m})$ \\
\hline 60 & 30 & 13 \\
85 & 43 & 23 \\
110 & 46 & 21 \\
140 & 54 & 20 \\
170 & 64 & 19 \\
200 & 68 & 19 \\
\hline
\end{tabular}

formation), and $\beta$ is the Coulomb friction angle. The mechanical values of $\tau_{y}$ and $R$ can be calculated by following the procedure described in Ref. [12].

The calculated results show that $\tau_{y}$ is $\sim 110 \mathrm{MPa}$ and $R$ is around $\sim 10 \mathrm{~J} / \mathrm{m}^{2}$ for the present PMMA at nanoscale cutting. It is notable that value of $R$ at nanoscale deformation and the theoretical free energy that required in creating new surfaces by breaking of covalent bonds $\left(\sim 1.5 \mathrm{~J} / \mathrm{m}^{2}\right)$ are found to be in the same order of magnitude. The macroscopic fracture energy $G_{\mathrm{c}}$ is several orders of magnitude larger than $R$ of nanocutting. For instance, Wyeth and Atkins [13] obtained $G_{\mathrm{c}}=640 \mathrm{~J} / \mathrm{m}^{2}$ for microcutting of PMMA, and Patel et al. [14] found the value of $G_{\mathrm{c}}$ for PMMA is around $1 \mathrm{~kJ} / \mathrm{m}^{2}$ in macroscale cutting. This is because the size of the plastic zone in front of the crack tip is significantly restricted in nanocutting while it is much larger in macroscale deformation.

\subsection{Nanocutting with Varying $v_{0}$}

\subsubsection{Chip and Surface Morphologies}

Nanocutting was performed on PMMA with $v_{0}$ varying from 0.25 to $10.0 \mathrm{~mm} / \mathrm{s}$, at the thickness $t_{\mathrm{u}}=85 \mathrm{~nm}$. Figure 5 displays the macroscopic features of the PMMA chips that were made at $v_{0}=0.25$ and $10.0 \mathrm{~mm} / \mathrm{s}$. Similar to the results obtained in Fig. 2, the chips had integral shapes, indicating that the cutting process in this speed range was controlled by a ductile deformation mode. These chips were also associated with microcracks oriented in the transverse direction.

The values of chip thickness and shear plane angle as a function of the cutting speed are given in Fig. 6. It can be seen that the chip thickness increases monotonically as the cutting speed increases from 0.25 to $1.0 \mathrm{~mm} / \mathrm{s}$ and then decreases slightly, while the shear plane angle shows an opposite trend.
Fig. 5 Macroscopic appearances of formed PMMA chips, from [8]
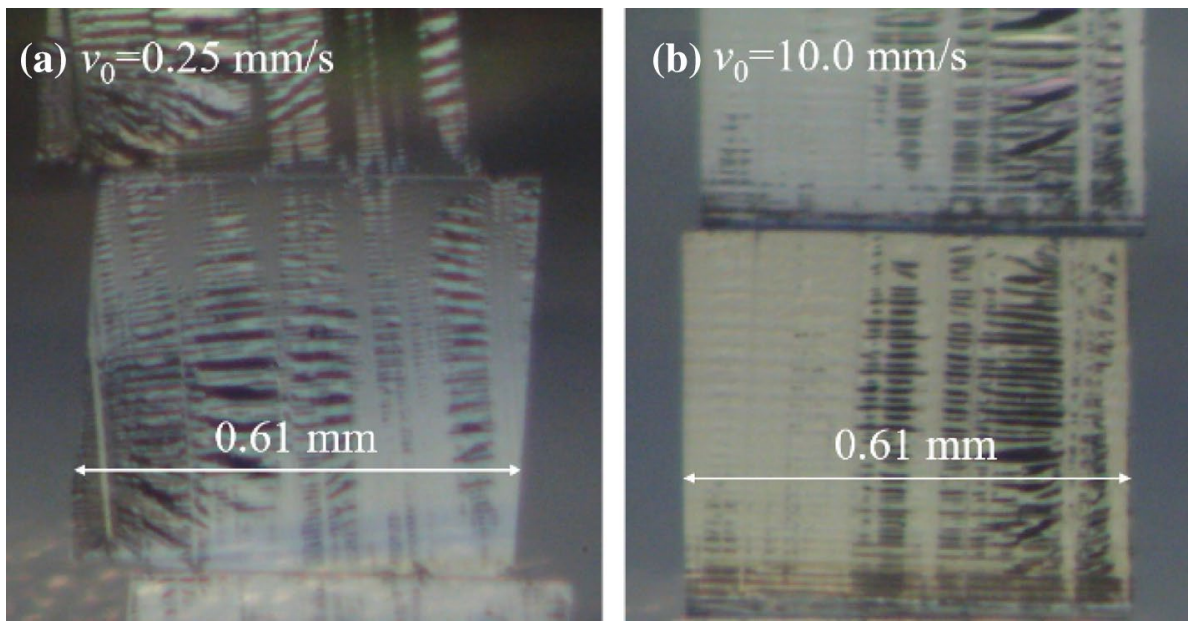


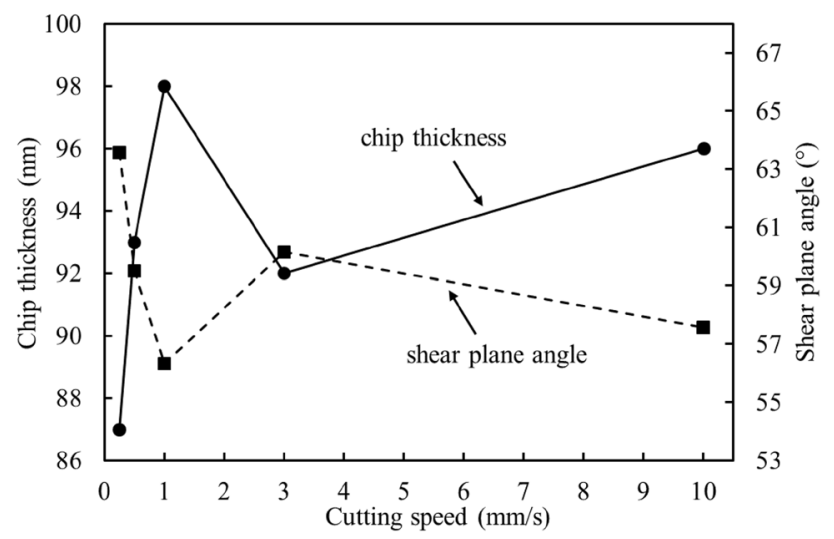

Fig. 6 Chip thickness and shear plane angle as a function of the cutting speed

The AFM morphologies of the cut surface of bulk PMMA made with varying speeds are displayed in Fig. 7. Below $v_{0}=1.0 \mathrm{~mm} / \mathrm{s}$, the surfaces were rather flat, while above this speed periodic structures oriented perpendicular to the cutting direction were formed. This can be observed from the plots of surface height profile along the cutting direction measured by AFM, shown in Fig. 8.

\subsubsection{Mechanical Response}

The measured values of the cutting force $F_{\mathrm{c}}$ and thrust force $F_{\mathrm{t}}$ are given in Table 3 . The cutting force $F_{\mathrm{c}}$ increases as $v_{0}$ increases from 0.5 to $1.0 \mathrm{~mm} / \mathrm{s}$ but decreases with further increase in cutting speed. It implies that the strain rate hardening due to the increasing cutting speed probably was diminished by thermal softening or damage mechanisms.

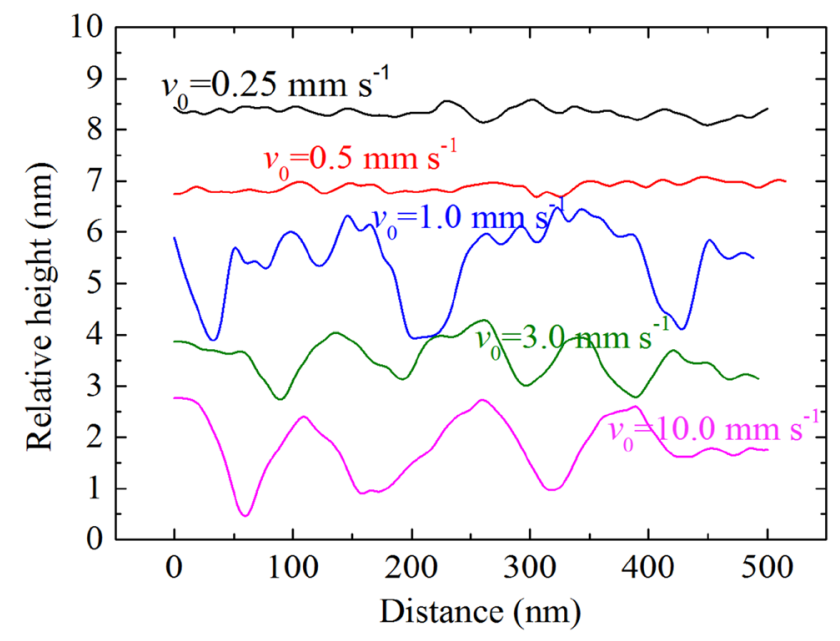

Fig. 8 Experimental surface heights along the cutting direction, from [8]

\section{Analysis and Discussions}

By comparing the surface morphology results shown above with findings in literature $[10,15]$, we attribute the periodic structures to the adiabatic shearing deformation mechanism during nanocutting. In this section, analytical and finite element modeling work including these physical mechanisms was conducted and compared with the experimental results.

\subsection{Adiabatic Shearing Modeling}

During plastic deformation, the increase in temperature has a negative effect on the material strength. If this effect overweighs the positive effect due to the strain rate hardening, a shear instability occurs [6]. Essentially, if the stress in the primary shear zone (PSZ), $\tau_{\mathrm{PSZ}}$, is surpassed by the stress in the bulk material, $\tau_{\text {Bulk }}$, a shear localization event takes place.
Fig. 7 AFM images of the cut surfaces created at $v_{0}=0.5$ and $3.0 \mathrm{~mm} / \mathrm{s}$, with arrows indicating the cutting directions
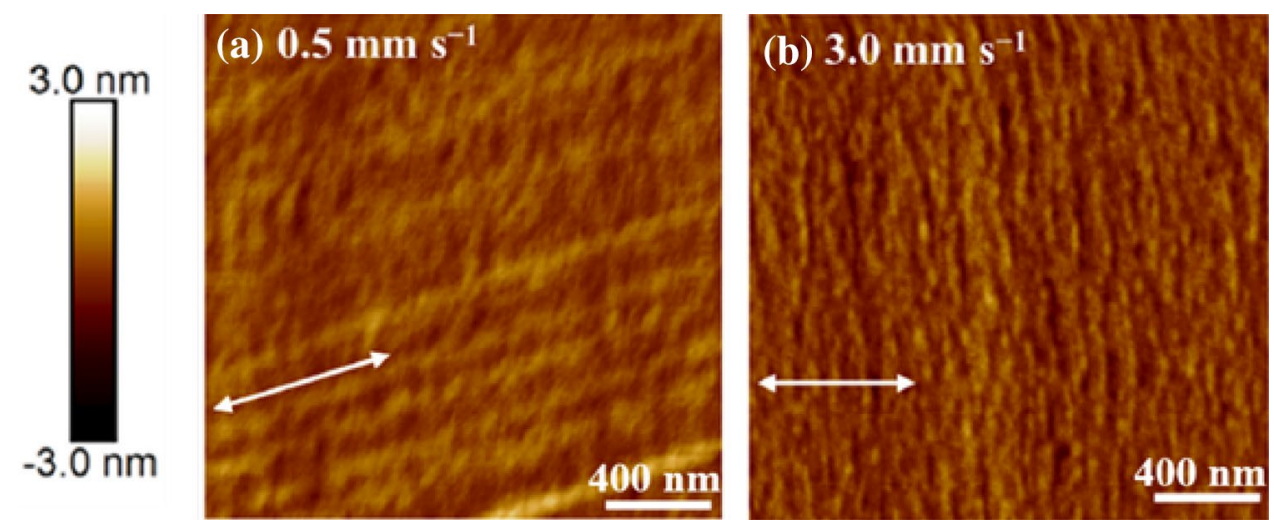
Table 3 Average values of cutting force and thrust force during nanocutting at different speeds, $t_{\mathrm{u}}=85 \mathrm{~nm}$

\begin{tabular}{llc}
\hline$v_{0}(\mathrm{~mm} / \mathrm{s})$ & $F_{\mathrm{c}} / w_{\mathrm{u}}(\mathrm{N} / \mathrm{m})$ & $F_{\mathrm{t}} / w_{\mathrm{u}}(\mathrm{N} / \mathrm{m})$ \\
\hline 0.25 & 30 & 4 \\
0.5 & 35 & 2 \\
1.0 & 42 & 4 \\
3.0 & 39 & 15 \\
10.0 & 37 & 63 \\
\hline
\end{tabular}

The effects of sectioning speed on temperature rise in the PSZ and in the bulk ahead were analyzed using Komanduri-Hou model $[15,16]$. A schematic drawing of the process zones of cutting in shear-banding material is found in Fig. 9. The model divides the formation of shear band into the shearing stage and flattening stage, and each includes two primary heating mechanisms. In the shearing stage, the plastic deformation in the PSZ and the friction on the interface between the chip segment already formed and the knife are the main heating mechanisms. In the flattening stage, the shearing on the second shear plane and the friction on the interface of the chip segment being formed and the knife are the other two primary heat sources. For instance, the temperature rise $\theta$ at an arbitrary position $M(x, y)$ due to the plastic deformation in the shearing stage is formulated in an integral form

$\theta_{M}=\frac{q_{0}}{2 \pi \lambda}\left(1-\frac{t}{2 t_{0}}\right) \int_{0}^{l_{s}} \Omega(p) \mathrm{d} y_{i}+\frac{q_{0}}{16 \pi \lambda \alpha_{t} t_{0}} \int_{0}^{l_{s}} r_{i}^{2} \chi(p) \mathrm{d} y_{i}$

where $q_{0}$ is the heat flux, $\lambda$ is the thermal conductivity, $t$ is the duration of the heat source, $t_{0}=l_{\mathrm{s}} v_{\mathrm{s}}\left(l_{\mathrm{s}}\right.$ is the length of the PSZ, $v_{\mathrm{s}}$ is the shear velocity on PSZ), $\alpha_{\mathrm{t}}$ is the thermal diffusivity, and

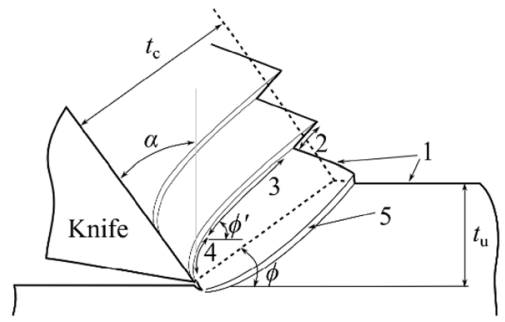

Fig. 9 Schematic illustration of the shear localization during cutting: (1) undeformed surfaces, (2) part of the shear failed surface separated from the following segment due to intense shear, (3) intense shear band formed due to shear in the flattening stage of the segment being formed, (4) intensely sheared surfaces of a segment in contact with the knife and subsequent slid on the tool face, and (5) intense localized deformation in the PSZ (Redrawn after Komanduri and Hou [15])
$\Omega(p)=\int_{p}^{\infty} \exp \left(-u^{2}\right) / u \mathrm{~d} u, \quad \chi(p)=\int_{p}^{\infty} \exp \left(-u^{2}\right) / u^{3} \mathrm{~d} u$

in which $p=r_{i} / \sqrt{ } 4 \alpha_{\mathrm{t}} t$ is a non-dimensional value and $r_{i}=\sqrt{ }\left[x^{2}+\left(y-y_{i}\right)^{2}\right]$ is the distance between the heat segment and the point $M(x, y)$.

As discussed in Sect. 1, the mechanical behavior of polymers is highly affected by the deformation rates, temperature change, etc. These effects need to be included in physical models describing the cutting processes in polymers. The yield stress of PMMA, $\sigma_{y}$, below the glass transition temperature is expressed as [3]

$\sigma_{y}=\sigma_{i}(0)-m T+\frac{2 k_{\mathrm{B}} T}{V_{\mathrm{a}}} \sinh ^{-1}\left(\frac{\dot{\varepsilon}}{\dot{\varepsilon}_{0} \exp \left(-\Delta H_{\beta} / k_{\mathrm{B}} T\right)}\right)^{1 / n}$

where $\sigma_{i}(0)$ is the athermal yield stress, $m$ is a softening parameter, $k_{\mathrm{B}}$ is the Boltzmann constant, $V_{\mathrm{a}}$ is the activation volume, $\dot{\varepsilon}$ is the strain rate, $\dot{\varepsilon}_{0}$ is a pre-exponential constant, $\Delta H_{\beta}$ is the activation energy, and $n$ is a molecular chain parameter. The values of the parameters in Eq. (5) can be found in Richeton et al. [3].

The temperature and yield stress in the primary shear zone (PSZ) and in the bulk ahead of the PSZ were calculated separately (please refer to [8] for more details). Predictions show that the onset speed for shear banding in PMMA nanocutting is $4-5 \mathrm{~mm} / \mathrm{s}$, above which the yield stress in the PSZ was exceeded by the stress in the bulk (cf. Figure 10), and the shear banding takes place. The predicted critical speed for the onset of shear band generally agrees with the experimental result of $\sim 1 \mathrm{~mm} / \mathrm{s}$ which is identified from Fig. 8 .

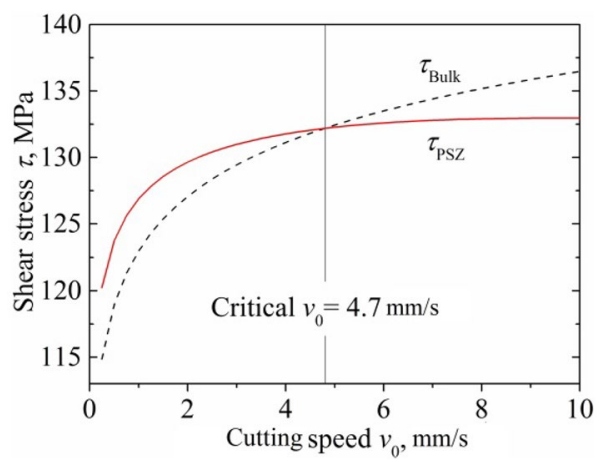

Fig. 10 Shear stresses in the primary shear zone and in the bulk material as a function of the cutting speed, from [8] 


\subsection{Finite Element Analysis}

Strictly, molecular dynamic (MD) method is more suitable for the nanocutting simulation than the finite element method considering the strong interplay among atoms and molecules under nanoscale deformation $[17,18]$; however, the MD method can only model a rather small volume cutting due to its computational limits. Therefore, the commercial finite element code Abaqus/Explicit (version 2017) is chosen to investigate the nanocutting of PMMA. In lack of experimentally characterized constitutive relations of the present PMMA, the experimental stress-strain relationship already including the effect of adiabatic softening by Richeton et al. [3] was adopted (please refer to the original Fig. 2 in [3]) to describe the yield and postyield behavior of PMMA. Figure 11 shows the 2D model for the cutting simulation. The CPE4R (4-node quadratic plane strain element with reduced integration) type elements were used for the chip and work material in the analysis. Pre-distorted meshes were generated in the chip zone to avoid severe element distortion during cutting [19]. The material separation between the chip and work material in front of the knife was achieved using a predefined cutting plane (a layer of 4-node elements) that joins the chip and work material parts. In the cutting plane, the built-in Abaqus shear damage model [20] was used and the material would degrade linearly (maximum failure displacement was equal to the size of the knife tip of $10 \mathrm{~nm}$ ) after the equivalent strain reached the critical value of 0.5 determined according to [3]. A sharp diamond knife was assumed in the model considering the radius of the diamond knife tip is only few nanometers. It was assumed that there was no friction between the chip and knife for ultramicrotome cutting.

In this simulation, the PMMA cutting at the thicknesses $t_{\mathrm{u}}=85 \mathrm{~nm}$ and $t_{\mathrm{u}}=200 \mathrm{~nm}$ was implemented, respectively, with the cutting speeds $v_{0}$ varying from 0.01 to $1.0 \mathrm{~mm} / \mathrm{s}$. In the case of $t_{\mathrm{u}}=85 \mathrm{~nm}$, a total of 20 elements were assigned along the chip thickness. In the case

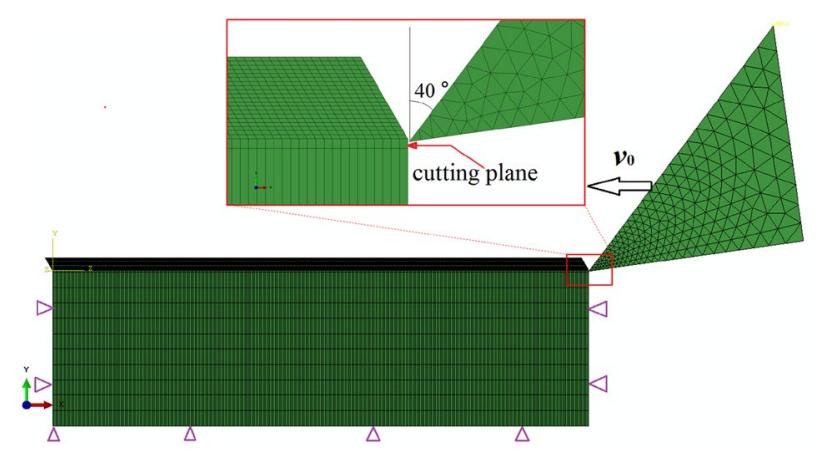

Fig. 11 2D model used in the cutting simulation of $t_{\mathrm{u}}=200 \mathrm{~nm}, 40$ elements were applied to have a similar mesh size for both chip parts.

\subsection{1 $t_{\mathrm{u}}=85 \mathrm{~nm}$}

Figure 12 shows the equivalent plastic strain for the cutting at $t_{\mathrm{u}}=85 \mathrm{~nm}$ under different speeds. As indicated in Fig. 12a, a continuous chip forms at $v_{0}=0.01 \mathrm{~mm} / \mathrm{s}$. The backside of the chip undergoes more plastic deformation than the front side due to the chip-knife contact and material separation mechanisms. As the cutting speed increases to $v_{0}=0.1 \mathrm{~mm} / \mathrm{s}$, localized deformation takes place as shown in Fig. 12b. Intense plastic strain develops near the knife tip and propagates to the free surface of the chip. As a result, an inclined shear band forms in the chip, leading to a serrated chip profile. Under a larger cutting speed, e.g., $v_{0}=1.0 \mathrm{~mm} / \mathrm{s}$ in Fig. 12c, localized deformation takes place as well, and the spacing between the adjacent shear bands decreases compared to the cutting result in Fig. 12b. During the formation of a shear band, localized plastic deformation takes place on the cut surface of the work material simultaneously, as displayed in Fig. 12b, c. The localized plastic deformation on the cut surface probably corresponds to the observed periodic patterns in experiment (Fig. 7b). According to [21, 22], a crack forms on the cut surface as a shear band generates in the chip. Since a pre-cutting plane method is used in the simulation, these cracks could not be predicted using this method.

The simulation reveals that the deformation in the chip transmits from a rather homogeneous mode to a localized mode as the cutting speed increases from 0.01 to $1.0 \mathrm{~mm} / \mathrm{s}$. As a result, the shape of the chip transmits from a continuous profile to a serrated one (localized shape). The predicted value of the critical speed for the onset of shear band is not discussed in this study since it can be easily obtained by a parametric study. Apparently, the value is much smaller than the experimental value of $1.0 \mathrm{~mm} / \mathrm{s}$.

\subsection{2 $t_{\mathrm{u}}=200 \mathrm{~nm}$}

Figure 13 shows the equivalent plastic strain for the cutting at $t_{\mathrm{u}}=200 \mathrm{~nm}$ under different speeds. Similar to the results in Fig. 12, continuous chip is obtained at $v_{0}=0.01 \mathrm{~mm} / \mathrm{s}$ and serrated chips are formed as the cutting speed increases.

Comparing Fig. 13b with Fig. 12b as well as Fig. 13c with Fig. 12c, which were cut at identical speeds, we can find that the shear bands in a thicker cutting (Fig. 13, $t_{\mathrm{u}}=200 \mathrm{~nm}$ ) have more intense plastic deformation and a smaller average spacing than those in a relative thinner cutting (Fig. 12, $t_{\mathrm{u}}=85 \mathrm{~nm}$ ). It implies that the critical speed for the onset of localized deformation decreases as the cutting thickness increases. 
Fig. 12 Simulated plastic strains in PMMA cutting at a cutting thickness $t_{\mathrm{u}}=85 \mathrm{~nm}$ under different cutting speeds
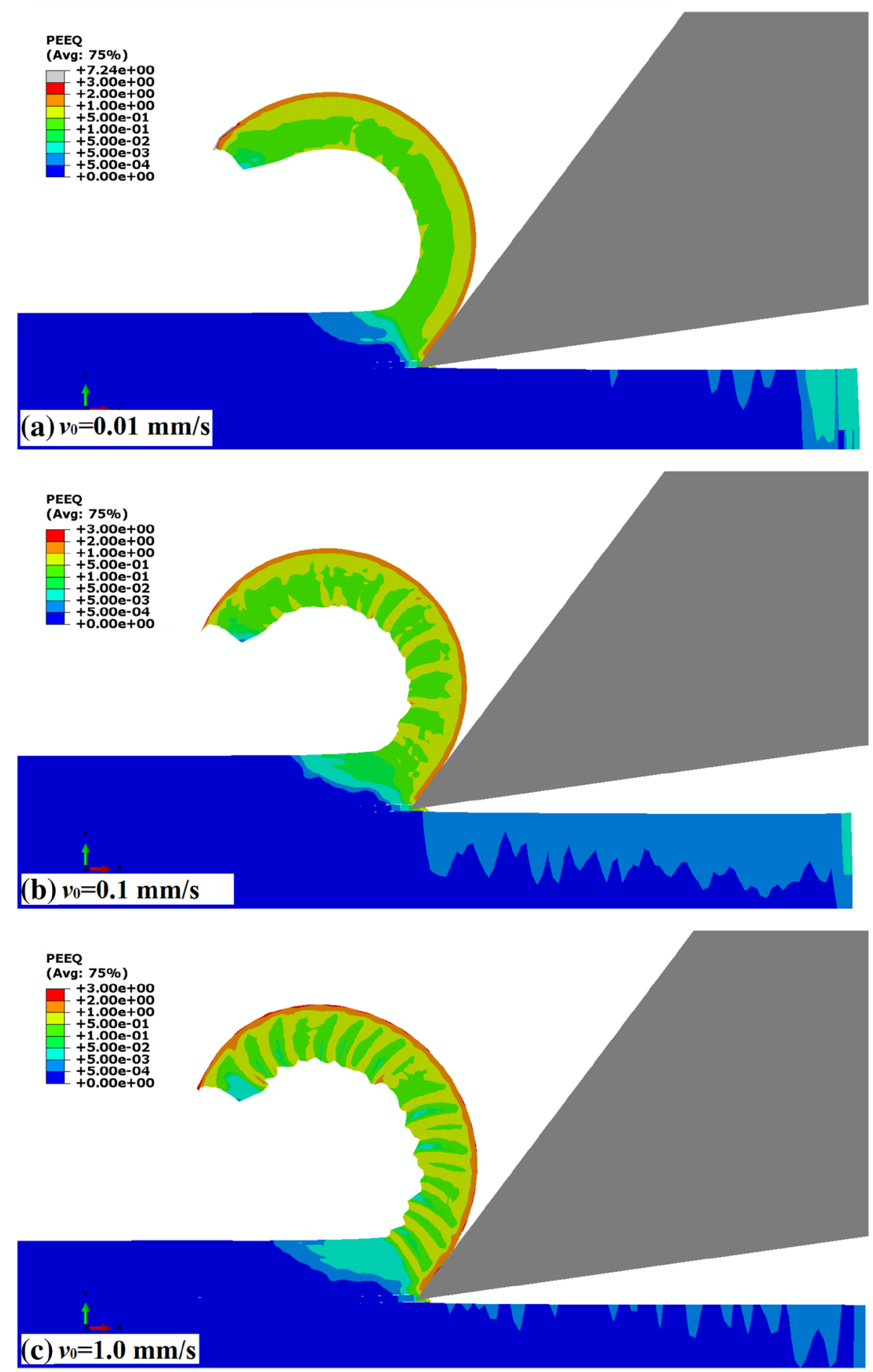

The above simulation results confirm that there is a transition of the deformation mode in the chip as the cutting speed (or thickness) varies, although the simulation gives a much smaller critical cutting speed than the experiment.

\subsubsection{Element-Type Sensitivity}

In the above simulation models, the chip parts use inclined 4-node quadratic plane strain elements, CPE4R, which would facilitate the formation of shear bands during cutting process. To investigate the mesh-type sensitivities of the 
Fig. 13 Simulated plastic strains in PMMA cutting at a cutting thickness $t_{\mathrm{u}}=200 \mathrm{~nm}$ under different cutting speeds
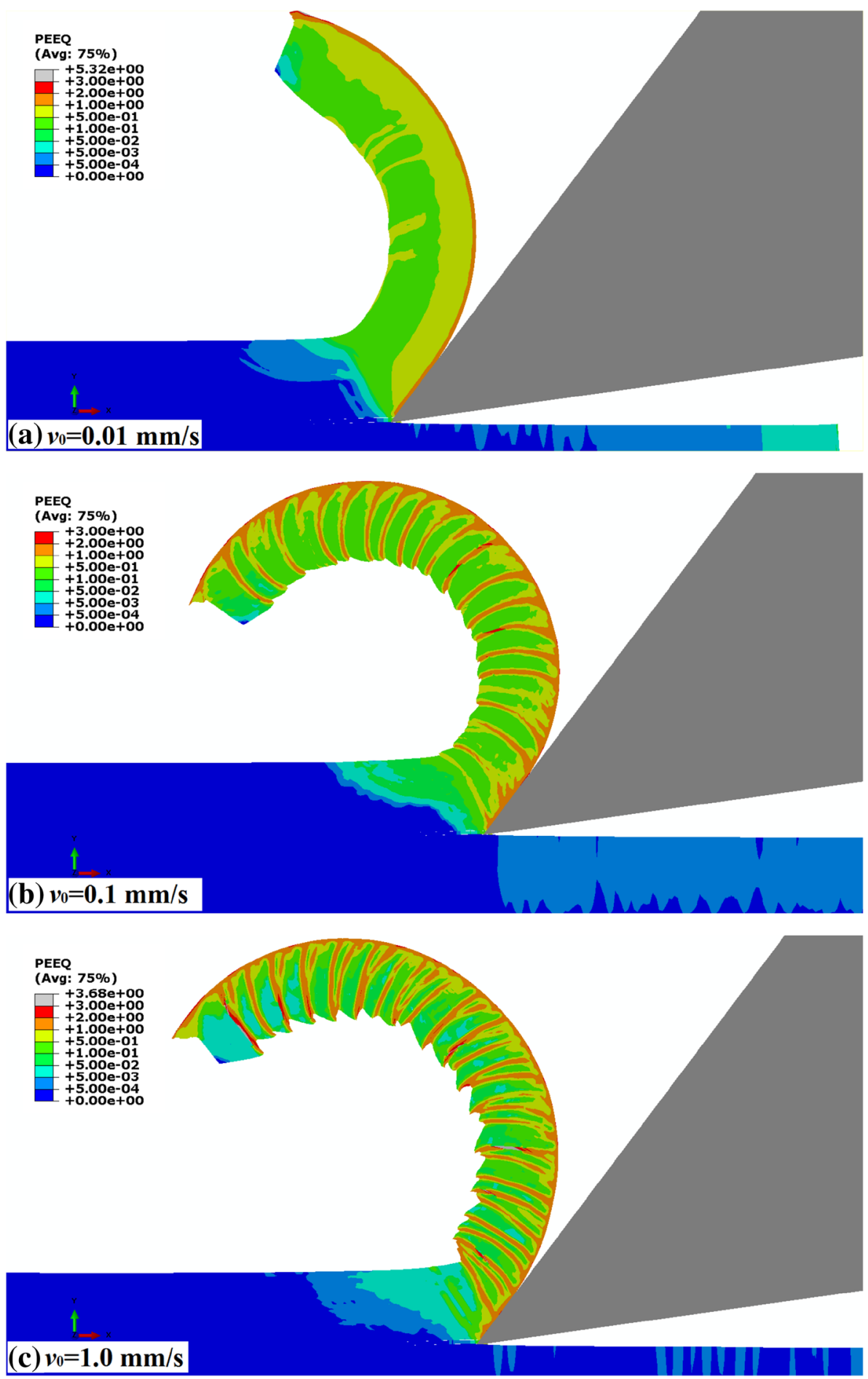

simulation, the quadratic elements were replaced by triangle plane strain elements, CPE3, in the chip part meshing.

Figure 14 displays the simulation results of cutting at $t_{\mathrm{u}}=85 \mathrm{~nm}$ under the speeds of 0.1 and $1.0 \mathrm{~mm} / \mathrm{s}$. Compared to the simulation results using quadratic elements shown in
Fig. 12, the cutting process is more steady as the cutting speed increases. The chips undergo more homogeneous deformation than those using quadratic elements and the chip profile becomes more continuous, but localized deformation still occurs. The results in Fig. 14 indicate that the PMMA cutting 
Fig. 14 Simulated plastic strains in PMMA using triangle plane strain elements, at a cutting thickness $t_{\mathrm{u}}=85 \mathrm{~nm}$
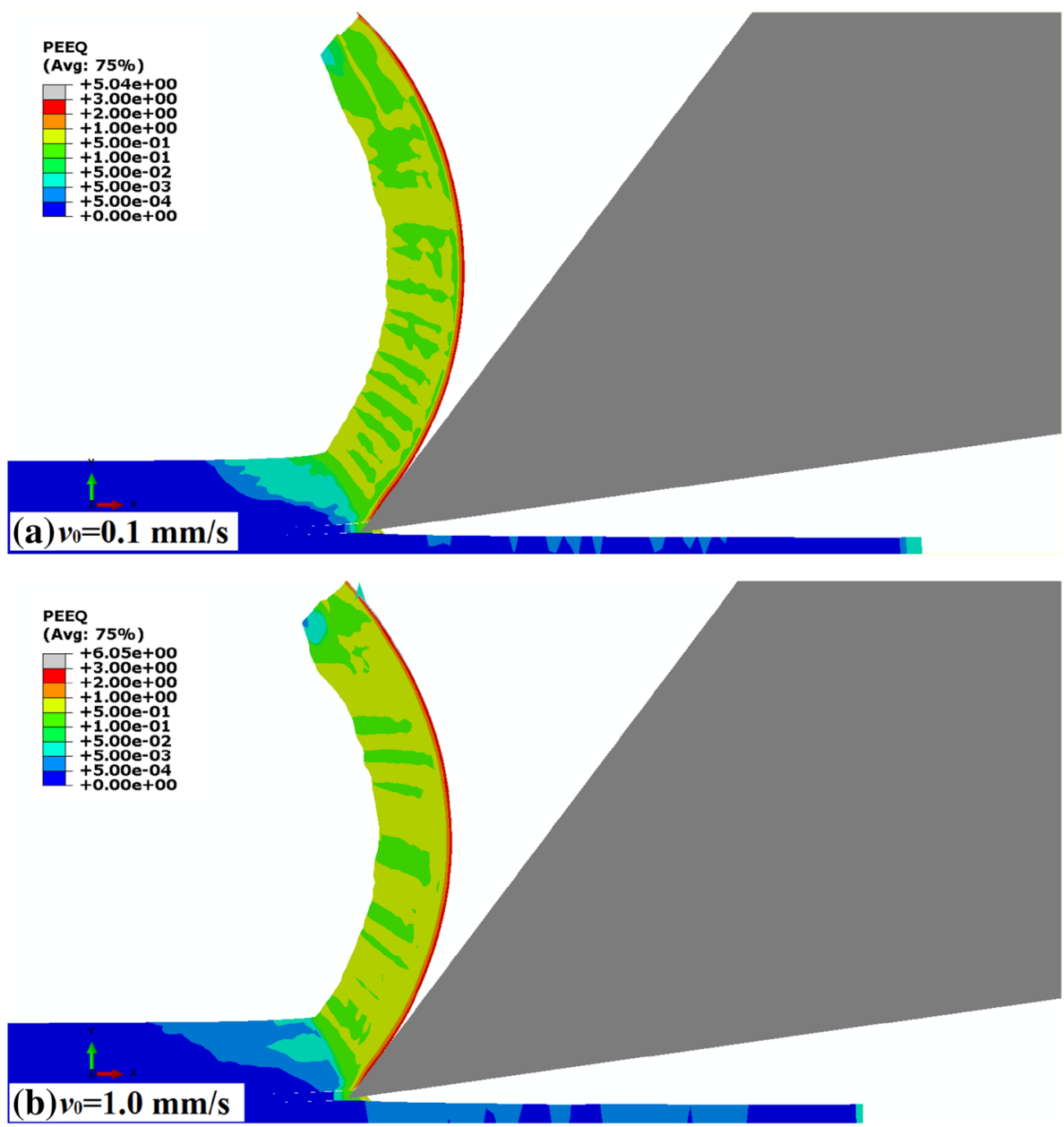

simulation is sensitive to the element type, which needs to take into account in the numerical analysis of PMMA cutting.

\section{Conclusions}

Periodic, wavy structures were found to form on the cut surface of PMMA at/above critical cutting thickness and speed. Below the critical cutting conditions, cut surfaces were flat and smooth, while above which periodic wavy structures formed. These wavy structures were identified as shear localizations originated from the yield deformation in the primary shear plane. The analytical modeling of the stress variation in the primary shear zone and in the bulk material in front of the shear zone predicts a critical speed for the onset of shear bands in cutting, which is relatively close to the experimental result of nanocutting. The finite element analysis also confirms that shear bands are formed when critical cutting conditions are reached.
Acknowledgements China Scholarship Council is acknowledged for the financial support. Dr. H. Li from Uppsala University is gratefully acknowledged for valuable discussions and experiment help.

Open Access This article is distributed under the terms of the Creative Commons Attribution 4.0 International License (http://creativeco mmons.org/licenses/by/4.0/), which permits unrestricted use, distribution, and reproduction in any medium, provided you give appropriate credit to the original author(s) and the source, provide a link to the Creative Commons license, and indicate if changes were made.

\section{References}

1. Rhorer RL, Evans CJ (2010) Fabrication of optics by diamond turning. In: Bass M (ed) Handbook of optics, 3rd edn. McGraw Hill, New York

2. Atkins AG (2016) Slice-push, formation of grooves and the scale effect in cutting. Interface Focus 6:20160019

3. Richeton J, Ahzi S, Vecchio KS, Jiang FC, Adharapurapu RR (2006) Influence of temperature and strain rate on the mechanical behavior of three amorphous polymers: characterization and modeling of the compressive yield stress. Int J Solids Struct 43:2318-2335 
4. Roesler J, Harders H, Baeker M (2007) Mechanical behaviour of engineering materials. Springer, New York

5. Dodd B, Bai Y (2012) Adiabatic shear localization-frontiers and advances, 2nd edn. Elsevier, Amsterdam

6. Recht RF (1964) Catastrophic thermoplastic shear. J Appl Mech 31:189-193

7. Sun F, Li H, Lindberg H, Leifer K, Gamstedt EK (2017) Polymer fracture and deformation during nanosectioning in an ultramicrotome. Eng Fract Mech 182:595-606

8. Sun F, Li H, Leifer K, Gamstedt EK (2017) Rate effects on localized shear deformation during nanosectioning of an amorphous thermoplastic polymer. Int J Solids Struct 129:40-48

9. Williams JG, Patel Y (2016) Fundamentals of cutting. Interface Focus 6:20150108

10. Burns TJ, Davies MA (2002) On repeated adiabatic shear band formation during high-speed machining. Int J Plast 18:487-506

11. Gong Y, Baik Y-J, Li CP, Byon C, Park JM, Ko TJ (2017) Experimental and modeling investigation on machined surfaces of HDPE-MWCNT polymer nanocomposite. Int J Adv Manuf Technol 88:879-885

12. Atkins AG (2003) Modelling metal cutting using modern ductile fracture mechanics: quantitative explanations for some longstanding problems. Int J Mech Sci 45:373-396

13. Wyeth DJ, Atkins AG (2009) Mixed mode fracture toughness as a separation parameter when cutting polymers. Eng Fract Mech 76:2690-2697

14. Patel Y, Blackman BRK, Williams JG (2009) Determining fracture toughness from cutting tests on polymers. Eng Fract Mech 76:2711-2730

15. Komanduri R, Hou Z-B (2002) On thermoplastic shear instability in the machining of a titanium alloy (Ti-6Al-4V). Metall Mater Trans A 33:2995-3010

16. Hou ZB, Komanduri R (1997) Modeling of thermomechanical shear instability in machining. Int J Mech Sci 39:1273-1314

17. Komanduri R, Chandrasekaran N, Raff LM (2000) MD simulation of nanometric cutting of single crystal aluminum-effect of crystal orientation and direction of cutting. Wear 242:60-88

18. Fang F, Xu F (2017) Recent advances in micro/nano-cutting: effect of tool edge and material properties. Nanomanuf Metrol 1:4-31

19. Vaz M Jr, Owen DRJ, Kalhori V, Lundblad M, Lindgren L-E (2007) Modelling and simulation of machining process. Arch Compt Methods Eng 14:173-204

20. Abaqus analysis user's manual 6.13, Dassault System
21. Turley DM, Doyle ED, Ramalingam S (1982) Calculation of shear strains in chip formation in titanium. Mater Sci Eng 55:45-48

22. Davies MA, Chou Y, Evans CJ (1996) On chip morphology, tool wear and cutting mechanics in finish hard turning. CIRP Ann Manuf Technol 45:77-82

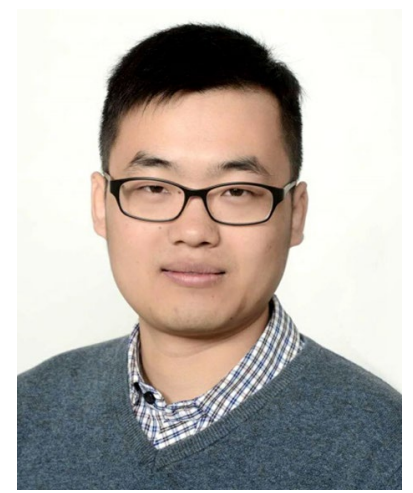

Fengzhen Sun research associate in the Department of Mechanical Engineering, Imperial College London, UK. He received his PhD degree from Uppsala University in 2017 . His recent work includes adhesive joining of metals, polymer nanocutting and fracture characterization.

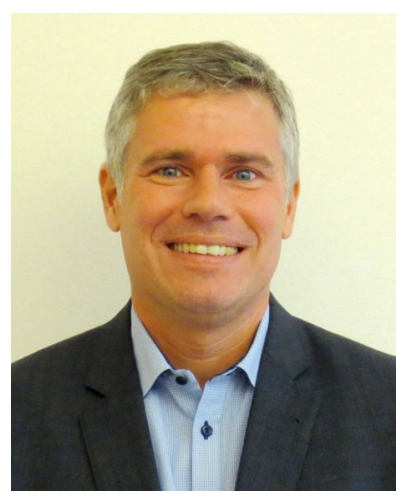

E. Kristofer Gamstedt professor in Applied Mechanics at Uppsala University, Sweden. His research interest includes the mechanics of composites, polymers, wood, biobased materials, biomaterials, coatings, etc. 